\title{
IV Conferencia Internacional de Turismo Sostenible Planeta, Personas, Paz (P3)
}

\section{Fourth International Conference on Sustainable Tourism Planet, People, Peace (P3)}

\author{
Mercedes Montero (MONTERO, M.) ${ }^{*}$ e \\ Monica de Nazaré Ferreira de Araujo (ARAÚJO, M. N. F.) ${ }^{* *}$
}

RESUMEN - El texto relata los principales puntos de discusión presentados en la IV Conferencia Internacional de Turismo Sostenible Planeta, Personas, Paz; con énfasis en los desafíos que presenta el desarrollo del turismo desde una perspectiva de sostenibilidad. Se presenta también la estructura y concepto del evento.

Palabras clave: Conferencia; Turismo Sostenible; Desafíos.

ABSTRACT - The following text recounts the main discussions presented in the IV International Conference on Sustainable Tourism Planet, People, Peace; emphasizing in the challenges of tourism development from a sustainability perspective. It also provides the structure and concept of the event.

Key words: Conference, Sustainable Tourism; Challenges.

\footnotetext{
* Formação: Economista Agrícola, Universidad de Costa Rica; M.Sc. International Agribusiness, Universität Göttingen. Estudiante de doctorado, Centro Agronómico Tropical de Investigación y Enseñanza (CATIE). Apdo. 7170 CATIE, Turrialba Cartago, Costa Rica. Telefone: (506)83096967. Email: montero@catie.ac.cr,mmonterovega@gmail.com.

** Formação: Licenciatura em Pedagogia (UNAMA), Graduação (Bacharelado) em Turismo (UFPA), Mestrado em Ciências da Comunicação (USP), Doutoranda do Núcleo de Altos Estudos da Amazônia (NAEA/UFPA). Bolsista da Coordenação de Aperfeiçoamento de Pessoal de Nível Superior/CAPES/BRASIL. Atividade profissional: Professora da Universidade Federal do Maranhão (UFMA). Endereço físico para correspondencia: Avenida dos Portugueses 1966, Departamento de Turismo e Hotelaria. CEP: 65080-805 - São Luís - Maranhão (Brasil). Telefone: 5598 3272-8441. Email: monicadenazare@gmail.com
} 
Informe de la Conferencia

4 a 6 de Noviembre, 2013

San José - Costa Rica

La IV Conferencia Internacional de Turismo Sostenible estuvo organizada por la Cámara Nacional de Ecoturismo y Turismo Sostenible de Costa Rica (CANAECO) y se llevó a cabo en el Auditorio Nacional del Museo de los Niños, en San José, con el objetivo de reunir y compartir las principales experiencias de líderes tanto nacionales (Costa Rica) como internacionales (Australia, Brasil, Bután, China, Colombia, Ecuador, Estados Unidos, y Kenia). Se consideraron temas como las buenas prácticas, desafíos y posibilidades del turismo sostenible en el mundo. El evento contó con las presencia de 193 participantes y tuvo como público a profesionales del campo, empresas, organizaciones gubernamentales y no gubernamentales, investigadores y estudiantes del área de turismo.

El evento se estructuró en cuatro áreas temáticas, tomando como referencia los elementos básicos de la existencia humana: agua, tierra, fuego y aire y sus asociaciones con el turismo sostenible y las tendencias actuales. Tuvo como eje transversal la motivación y la innovación que posibilitó a los asistentes un autoanálisis como personas y el enriquecimiento de sus actitudes y oportunidades para enfrentar el desafío de un mundo cada vez más cambiante. Además de las presentaciones, los asistentes pudieron participar en giras de campo a empresas y proyectos de turismo con prácticas sostenibles: Chocolate Sibú, Finca Rosa Blanca Coffee Plantation \& Inn, El encanto de la Piedra Blanca, Hotel Holiday Inn Escazú y Mapache Rent a Car.

La sesión de inauguración de estuvo a cargo de varias personas: inició con un mensaje de bienvenida que estuvo a cargo de Ana Báez ${ }^{1}$; Glenn Jampol ${ }^{2}$ presentó a CANAECO, los objetivos del evento y su importancia para el avance las prácticas de turismo en Costa Rica y en el mundo; Gustavo Segura ${ }^{3}$ enfatizo en las cuatro dimensiones del turismo: la investigación científica, los aspectos sociales, la economía y

\footnotetext{
${ }^{1}$ Vicepresidenta de CANECO y Maestra de Ceremonias de la conferencia.

${ }^{2}$ Presidente de la Cámara Nacional de Ecoturismo y Turismo Sostenible de Costa Rica.

${ }^{3}$ Vicepresidente de la Junta Directiva del instituto Costarricense de Turismo (ICT).
} 
la capacidad empresarial; Laura Chinchilla ${ }^{4}$ envió un mensaje virtual habló sobre los esfuerzos de Costa Rica en el área de turismo y finalmente se presentó Ana Clara Carranza con una motivación llamada La Historia del Colibrí. La exposición principal de este bloque "El turismo toma nuevos caminos" estuvo a cargo de Elizabeth Baker" quien enfatizó en el movimiento del turismo mundial en términos económicos y sus efectos en el mercado laboral, asegurando que el turismo en las actividades turísticas más poderosas en el mundo, sin embargo este poder no se está utilizando. Esa conclusión se basa en un viaje de 5 años alrededor del mundo que Becker realizó para verificar cómo los países tratan el turismo como uno de los negocios más significativos para sus economías.

El primer bloque de presentaciones del evento titulado "Agua" trató de las economías y de cómo cada sector debería aumentar la inversión en sostenibilidad con el objetivo de generar mayores retornos en el ámbito económico, ambiental y social. Laksmi Tamara Budowski ${ }^{6}$ presentó la charla de motivación titulada "Preparándonos para el cambio" enfatizando en que hoy día existe un despertar mundial de la búsqueda de la paz interior y que Costa Rica se ha convertido en un destino importante de paz. El Shamanismo constituye una tendencia de turismo porque las personas están viviendo en un mundo con una serie de factores de orden político (guerras, ingobernabilidad y enfrentamientos armados), económico (crisis estadounidense), ambiental (cambio climático) y social (altas tasas de divorcios) que provocan un acelerado modo de vida cotidiano que ha generado que la depresión, ansiedad y desesperanza sean parte de la rutina de los seres humanos. Costa Rica ha sido un destino más resistente a la crisis mundial y podría inspirar al mundo pues es un país democrático, sin ejército, con un alto nivel educativo y espacios naturales de gran belleza, compatible con la práctica del ecoturismo, turismo de aventura y turismo sostenible.

Seguidamente, la charla principal estuvo a cargo de Lawrence Pratt ${ }^{7}$ cuyo tema fue "Retornos sobre Inversiones en Sostenibilidad: imperativo en economía verde".

\footnotetext{
${ }^{4}$ Expresidenta de la república, periodo 2010-2014.

${ }^{5}$ Periodista y autora de Overbooked, USA.

${ }^{6}$ Directora de Operaciones Instituto Sat Yoga, de Costa Rica.

${ }^{7}$ Director del Centro Latinoamericano para la Competitividad y el Desarrollo Sostenible, INCAE, Costa Rica.
} 
Presentó los motivos para invertir en turismo sostenible: la ecologización del turismo reduciría el costo de la energía y el agua, aumentaría el valor de la diversidad, de los ecosistemas y de la herencia cultural, el turismo tiene potencial para crear nuevos y mejores empleos, el desarrollo turístico puede apropiar a las economías locales de herramientas de desarrollo y reducir la pobreza. Pratt concluyó su charla, asegurando que los turistas están exigiendo prácticas más sostenibles en el sector turístico y que las pequeñas empresas necesitan movilización y acceso a financiamiento para ofrecer un turismo más verde, tanto del sector turístico como de sus cadenas. El planeamiento de destinos y las estrategias de desarrollo son los primeros pasos para el desarrollo de la ecologización del turismo, además las políticas de inversión gubernamental pueden influenciar a los actores privados hacia este proceso.

Posteriormente, Julio Bin $^{8}$ y Gisela Sánchez ${ }^{9}$ presentaron el panel "Desarrollo de Negocios Sostenibles" y "Rentabilidad sí, pero calidad de vida también" respectivamente. El eje de la discusión del primer panelista se basó en la pregunta ¿Para quién trabaja usted, cuál es su meta, al final del año, fue producción de vida? Enfatizó en que los negocios sostenibles deberían estar trabajando para el planeta sustituyendo el negocio del lucro por el negocio de vida. Esto es una nueva percepción de vida en la que se involucra a la sociedad, el medio ambiente y la economía, es decir: como ciudadanos y profesionales se hace de la vida la base de todas nuestras acciones. De esta manera, sería necesario un nuevo modelo de negocios que integre las dimensiones sociales y ambientales en el crecimiento económico.

La segunda panelista, Gisela Sánchez, abordo los objetivos estratégicos, los proyectos y resultados de la empresa Florida Ice \& Farm Co (FIFCO) ${ }^{10}$ en las áreas: ambiental (Cero Residuos Sólidos 2011, Agua Neutral 2012, Carbono Neutral 2017), social (Consumo Inteligente, Inversión Social e Rendición de cuentas, Seguridad, Talento y Cultura) y económica (Liderazgo de Mercado, Crecimiento y Rentabilidad).

El segundo día de conferencias se titula bajo el eje temático del bloque "tierra" que enfatiza en los destinos sostenibles. Para iniciar con las charlas, Judy Kephner-

\footnotetext{
${ }^{8}$ Especialista en Negocios Sostenibles, Brasil.

${ }^{9}$ Directora de relaciones corporativas de Florida Ice \& Farm Co.

${ }^{10}$ Para mayor información ver < http://www.planetpeoplepeace.com/es/conferencias-anteriores/p3-2013>.
} 
Gona $^{11}$ propone una visión en la que la motivación hacia el turismo sostenible debe ser personal. Menciona la protección de las personas indígenas de los pueblos (y su vínculo con la naturaleza), la generación de un turismo basado en el orgullo nacional por medio de mejores leyes que promuevan los valores de sostenibilidad para finalmente generar un mundo mejor. Se habla de leadership counsiosness que es esa capacidad de reconocimiento propio de liderazgo, así como ejercerlo en un planeta que necesita más líderes en sostenibilidad. De esto se desprende los dos mayores retos para el turismo sostenible según la expositora que son: la creación de un grupo de líderes, ya que hay más seguidores que líderes y el segundo reto clave es que el sector turismo trabaje como grupo y que no sean solamente esfuerzos aislados. Si se logra mayor unidad, se tendría mayor impacto, derivado de un decir africano: "Si queremos ir rápido debemos ir solos, pero si lo que queremos es llegar lejos, debemos ir acompañados".

Seguidamente se presentó la experiencia turística de Bhután, que estuvo a cargo de Damcho Rinzin ${ }^{12}$ que presentó una propuesta turística alternativa en Bhutan como destino exclusivo para un grupo selecto de turistas en la que no se deja abierta la opción para turismo masivo. Rinzin plantea que si se quiere optar por un turismo realmente sostenible, hay que establecer límites y esto es lo que su país ha hecho, por medio de una política nacional de desarrollo turístico. El turismo en Bhután está altamente controlado: hay rutas pre-establecidas, lugares prohibidos y días establecidos para realizar las excursiones. Este es un caso de cómo gestionar el turismo basado en responsabilidad, autenticidad, calidad y unicidad que son los cuatro pilares de la política de turismo del país.

Posteriormente se presentó la charla de Amos Bien ${ }^{13}$ quien realizó una exposición sobre los criterios de turismo sostenible del Global Sustainable Tourism Council (GSTC). Él hace referencia a que no hay un entendimiento común del término de Turismo Sostenible, sin embargo los criterios presentados proponen una solución operativa y que el reto ha sido poder generar criterios universales para destinos porque implica incluir en el análisis la generación de ventajas para las comunidades. El GSTC

${ }_{11}$ Directora Ejecutiva de Kenya Conservation Land Trust (KLCT). Información disponible en: $<$ http://www.klct.or.ke/>.

12 Tourism Council Bhutan.

13 Consultor Principal: Global Sustainable Tourism Council. Información disponible en: <http://www.gstcouncil.org/>. 
ha desarrollado 4 objetivos con 41 criterios y 100 indicadores basados en: la gestión sostenible, maximización de los beneficios sociales, comunales, a los visitantes y patrimonio, así como la maximización de los beneficios al medio ambiente.

Seguidamente se presentaron estudios de caso de diferentes localidades que han presentado un gran desarrollo turístico dentro del marco de la sostenibilidad. La primera charla de este bloque la presentó Sergio Moreno ${ }^{14}$ cuya exposición se basó en la Isla de Lanzarote como destino turístico. Primeramente comentó acerca de las claves de éxito del turismo en Lanzarote, dadas las particularidades de esta región. El primer punto para obtener éxito es la conciencia social: la naturaleza, el arte, el desarrollo y la sociedad se han involucrado conjuntamente y se ven reflejados en las políticas públicas de promoción en inclusión. El segundo punto ha sido el de preservar por transformación. Por definición, Lanzarote no es un destino verde, por lo que para la promoción del destino se han adaptado varias modalidades de transformación y adaptación del paisaje que no son las típicas, dándole un valor agregado a cada micro-cluster. Es por esto que se han diseñado planes vacacionales adaptados, ya que la política de desarrollo turística se ha volcado hacia la especialización y diferenciación de destinos dentro de Lanzarote.

A continuación, Carlos Albán ${ }^{15}$, representante de Perú, presentó el desarrollo del turismo en este país, cuya economía se ha visto fuertemente impulsada por este sector en los últimos años. Se cree que este aumento se debe a: el crecimiento económico, el crecimiento de la proporción de la clase media y la consistencia de la política institucional peruana. Se ha desarrollado un plan estratégico a nivel país en Perú para el desarrollo turístico que incluye la diversificación de la oferta turística, y la articulación público privada. Albán presenta un ejemplo usando The Travel and Tourism Competitiveness Index en el caso de Cusco. El proceso de evaluación consistió en la documentación y mapeo, la evaluación in situ, validación de la información y generación de un informe. Se encontró que las principales limitantes en la zona fueron: que no existe una gerencia integral, hay una baja voluntad política, falta de capacidad técnica, planes no incluyen el componente de la sostenibilidad, no existe un monitoreo de la contribución del turismo y faltan códigos e índices para certificación. Dados estos

\footnotetext{
${ }^{14}$ Director Adjunto para el Instituto de Turismo y Desarrollo Económico Sostenible. Universidad Las Palmas de Gran Canaria, España.

${ }^{15}$ Director de la Dirección de Desarrollo Turístico de Perú.
} 
resultados y con base en el índice mencionado anteriormente, se generaron metas sociales, económicas y ambientales articulando la inclusión de la comunidad en el desarrollo del destino como estrategia de mejora.

Patricio Gaybor ${ }^{16}$ centró su presentación en la exposición de Quito, Ecuador, como un caso exitoso en la promoción de destinos turísticos. En la constitución ecuatoriana se incluye del desarrollo social, ambiental y económico como parte de los derechos de los ecuatorianos y en la definición de desarrollo, por lo que las políticas gubernamentales han sido clave en el desarrollo turístico del país. Desde el 2008 se tomó la decisión de descentralizar el manejo turístico por lo que los gestores del turismo se encuentran ahora en cada localidad. Partiendo del supuesto de la necesidad de integración de las comunidades y los gobiernos locales como gestores y promotores de su propio desarrollo, los gestores de la promoción turística de Quito generaron una micro-planificación para gestionar el atractivo turístico desde la perspectiva del centro histórico mejor conservado de América Latina. La promoción de Quito como destino turístico ha tenido gran acogida y se considera un caso de éxito dentro de la propuesta ecuatoriana de micro-planificación.

Rodolfo Lizano ${ }^{17}$, como representante del Instituto Costarricense de Turismo (ICT) realizó una reseña histórica de las acciones que han tomado los diferentes actores involucrados en el turismo nacional. Con respecto a la sostenibilidad del sistema deja muy en claro que la sostenibilidad no es algo que se da, sino que hay que gestionarla. En Costa Rica se han desarrollado dos mecanismos de gestión por medio de certificaciones: La CST y la Bandera Azul. Sin embargo estos esfuerzos requieren de una promoción en los deseos del turista, ya que el mercado funciona de acuerdo con los deseos de este. Uno de los aspectos más importantes de la sostenibilidad turística es la consideración de las restricciones regionales y del involucramiento regional en la experiencia turística. A pesar de la buena experiencia costarricense por la promoción del turismo sostenible, sigue existiendo un gran paradigma entre la sostenibilidad y el libre mercado, la globalización económica y el movimiento económico.

\footnotetext{
${ }^{16}$ Gerente Técnico, Quito Turismo, Ecuador.

${ }^{17}$ Director de Planeamiento y Desarrollo Turístico, ICT.
} 
Para empezar con el siguiente bloque "aire" que correspondía a la marca país, Andrey Elizondo ${ }^{18}$ empezó la sección con una charla motivacional en la que se hizo énfasis en el ecoturismo y la innovación en Costa Rica donde destaca que la adopción temprana de políticas de sostenibilidad es la plataforma para lo que el país tiene hoy en día.

Como charla principal, se contó con la participación de Mariella Soldi ${ }^{19}$ exponiendo acerca del éxito que ha tenido la marca país en Perú y cómo se ha ido gestionando su posicionamiento. Soldí comenta sobre cómo cuando diferentes grupos independientes gestionaban diferentes marcas del país realmente no había una sinergia hacia la obtención de una marca reconocida y representante de la esencia peruana. La creación de la marca Perú nace en el año 2008 y el éxito de la estrategia de posicionamiento ha sido que esta ha sido creíble, relevante y diferenciada.

Posteriormente se presentó un bloque de estudios de casos de diversos países que han incorporado de alguna u otra manera una marca país o regional. Como primer expositor estuvo León Trujillo ${ }^{20}$ refiriéndose a la creación de marcas regionales y nacionales en Colombia. Principalmente enfatiza en que las marcas no pueden ser desechables y que se requiere de un proyecto a largo plazo, por lo que da 4 recomendaciones: 1. Hay que saber comunicar la estrategia, 2. Las marcas se construyen, no se imponen, 3. Se debe promocionar la vivencia de la experiencia, 4. Es preferible tener una política estatal que una gubernamental para que esta tenga continuidad.

Tony Charters ${ }^{21}$ enfatizó su charla en la promoción y desarrollo turístico en Nueva Zelanda. El turismo es la segunda actividad con respecto al aporte al Producto Interno Bruto y el $25 \%$ de este proviene del ecoturismo. Se dice que estos alcances se han logrado gracias a la creación de una marca que refleja escasez y unicidad. A pesar de los problemas que enfrenta Nueva Zelanda (aislado, costoso, muy grande para visitar en un solo viaje), la promoción del país se ha mantenido fiel a la realidad del país por lo que el turismo se ha promocionado como turismo de alto nivel en el que se maneja el

\footnotetext{
${ }^{18}$ Investigador Asociado para INCAE, Costa Rica.

${ }^{19}$ Proyecto Marca País Promperu.

${ }^{20}$ Experto en Mercadeo y Branding Territorial.

${ }^{21}$ Director de Tony Charters y Asociados.
} 
componente de sostenibilidad. Sin embargo esta marca país debe ser creíble cuando el turista finalmente visita el destino. En un caso similar Inngun Sornes ${ }^{22}$ expuso sobre la experiencia de Noruega que como país se ha promocionado como un destino sostenible por medio de una estrategia planteada en el año 2012 en la que la naturaleza ha sido la principal atracción y fuente de inspiración para la creación de la marca país. La última charla de este bloque estuvo a cargo de Silvia Rodríguez ${ }^{23}$ representante del Instituto Costarricense de Turismo (ICT) en la que se presentó la nueva marca país de Costa Rica. La creación de la nueva marca se ha gestado por medio del ICT y de la Promotora de Comercio Procomer y se ha pasado del "Sin ingredientes artificiales" a "Essential Costa Rica" en un esfuerzo por promocionar al país desde una nueva perspectiva más inclusiva.

El bloque "fuego" estuvo guiado por el tema de cómo mercadear la sostenibilidad. La charla motivacional estuvo a cargo de Francisco Villalta ${ }^{24}$ que propuso una coherencia entre lo que una persona hace, cómo lo hace y para qué lo hace, para lograr alineamiento y paz en la vida de las personas.

La siguiente charla dentro de este bloque estuvo a cargo de Lance Barnett ${ }^{25}$, representante de Chiquita. En un mercado diferente del turismo, Barnett expuso las prácticas adoptadas por Chiquita para generar una agricultura más sostenible. Las claves del éxito para esta empresa se centran en la creación y medición de estándares así como la capacidad y motivación de su principal activo, que son sus empleados. La inclusión de los grupos de interés o stakeholders en el proceso hacia la sostenibilidad ha sido clave, especialmente la inclusión de las comunidades por medio de la educación en torno a la sostenibilidad y la conservación. Un punto importante de acuerdo con esta exposición fue el enunciado que lidera la campaña de Chiquita: mejora de la calidad de vida, amor al planeta y el liderazgo en esta industria.

\footnotetext{
${ }^{22}$ Asesora Especial Innovation Norway.

${ }^{23}$ Jefa de Promoción, ICT.

${ }^{24}$ Senior y Master Coach International.

${ }^{25}$ Director Global de Calidad. Chiquita Brands.
} 
Posteriormente Mei Zhang ${ }^{26}$ expuso los esfuerzos pioneros en China para diferenciarse aportando a la sostenibilidad por medio de una iniciativa privada. Contrario al turismo de masas, esta propuesta generada por el tour operador WildChina está diseñada para pequeños grupos que quieran visitar China dentro de un contexto de sostenibilidad que incluye desde el hospedaje hasta le selección de restaurantes locales que generen beneficios a las comunidades. Como última charla de este bloque se presentó a Hans Pfizer $^{27}$ que ha optado por la sostenibilidad como herramienta de diferenciación y generación de valor.

El siguiente y último bloque "viento" fue dedicado a una sesión de preguntas y respuestas de todos los participantes y expositores, donde se discutió de los principales temas abordados y políticas para el fomento del turismo sostenible. Cabe resaltar la importancia de las políticas públicas pero también el accionar individual de todas las personas para optar por un modo de vida más sostenible, tanto desde la empresa privada como las sociedades.

La IV edición de la Conferencia Internacional Turismo Sostenible Planeta, Personas, Paz - P3 recibió en marzo del 2014, el certificado del Programa Bandera Azul Ecológica, por su excelencia en la categoría de eventos especiales, siendo el primer evento turístico galardonado con este reconocimiento. Cumplió con los criterios establecidos de: racionalización de agua, energía, papel, acceso a agua potable, manejo adecuado de residuos sólidos y prácticas limpias en la preparación de alimentos. Cabe resaltar que toda la alimentación del evento fue $100 \%$ de origen vegetal y orgánico con productos provenientes de diversas zonas de Costa Rica.

\section{REFERENCIAS}

EVENTO especiales obtienen bandera azul por primera vez. Disponible en: <http://www.nacion.com/vivir/ambiente/Eventos-especiales-obtienen-primerabandera_0_1404459571.html>. Acceso en: 20/05/2014.

\footnotetext{
${ }^{26}$ Fundadora y CEO de Wild China.

${ }^{27}$ Propietario de la línea de hoteles de lujo Cayuga.
} 